\title{
AUTOMATED TRAFFIC MANAGEMENT SYSTEM USING BIG DATA TECHNOLOGY
}

\author{
N. Naga Lakshmi ${ }^{1}$ and T.Asha Latha ${ }^{2}$
}

Abstract: Generally Automation plays an important role in the global economy and in daily experience. The production of large amounts of data has not only been observed in web based companies but has seen entering other domains such as automation and automobile. Smart sensors, smart devices, closed circuit cameras, contribute to growing amounts of data that need to be processed. The outcome expected from processing is prediction for better traffic control. In existing system challenges at large scale performing large-scale computation is difficult and large amount of data is generated from sensors. This paper examines the utilization of big data technologies for traffic maintenance and performance domain. The approach is based on sensor measurements with the goal of detecting specific events and patterns.

Keywords - Prediction, Automation, patterns, smart sensors, computation, big data

\section{INTRODUCTION}

Generally Automation plays an important role in the global economy and in daily experience. Engineers strive to combine automated devices with mathematical and organizational tools to create complex systems for a rapidly expanding range of applications. The Automated traffic management system is an automated system that is used to manage traffic and its administration. It is meant to provide the administration and staff, with information in real time to make their work more interesting and less stressing.

In today's world data is generated from varied locations when compared to couple of years and all the data accumulated in past two years is more than for past 100 years. As we have the technology to generate, process, accumulate and store data. The normal data becomes a new framed technology of big data when it processes the four ' $\mathrm{V}$ ' properties.

${ }^{1}$ Department of Information Technology Anurag Group of Institutions, Hyderabad, Telangana, India

${ }^{2}$ Department of Information Technology Anurag Group of Institutions, Hyderabad, Telangana, India 


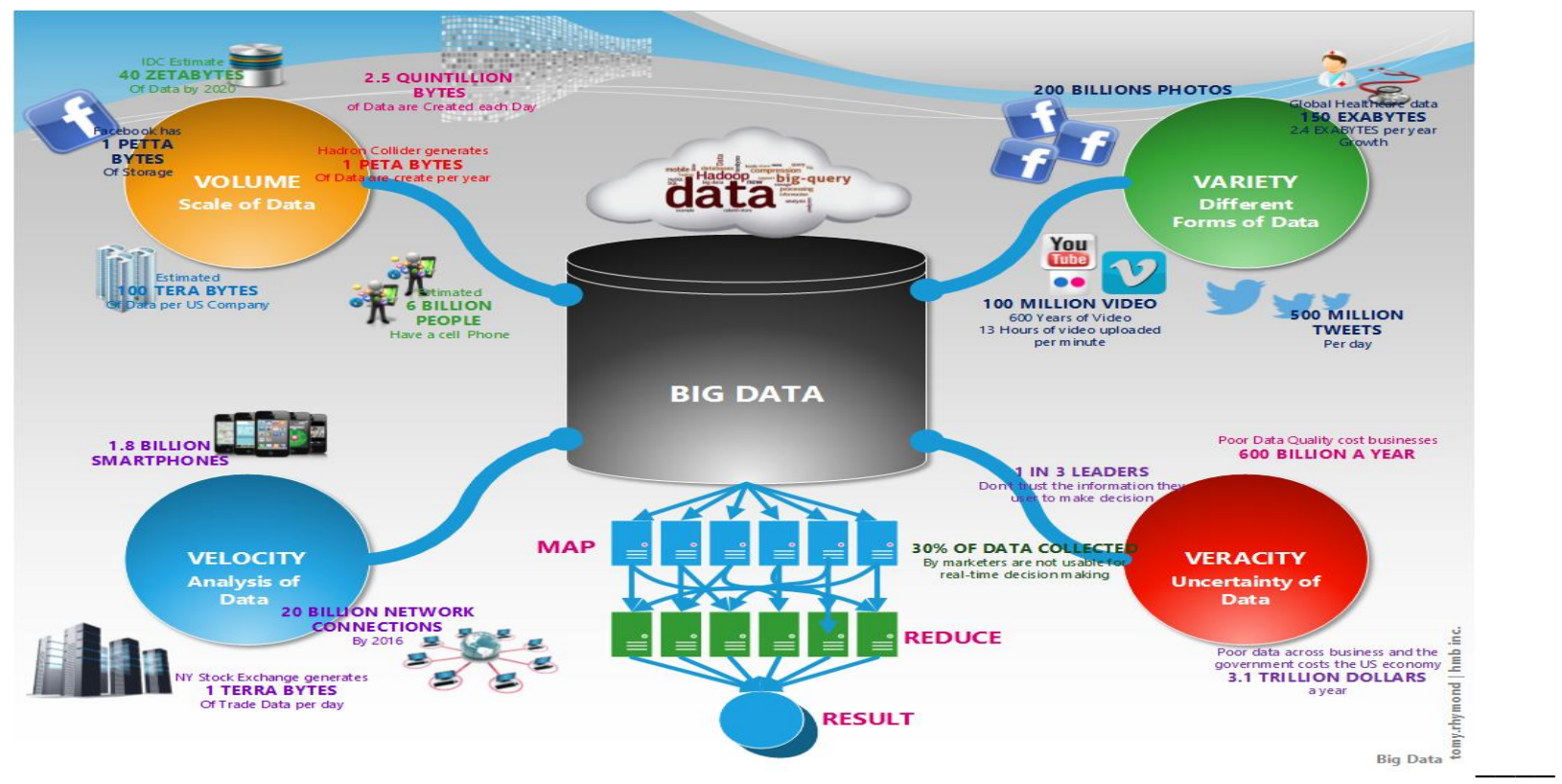

The data which multiplies quickly and it size limit crossing more than peta bytes is associated with 1st 'V-Volume'. The data movement is in very high speed network systems which is mostly applicable to all the real time scenarios of applications it's the 2nd 'V - Velocity'. The data generated from varied application posse's characteristic of usual formatted and structured data like excel sheets and database data within a file structure. The semi structured data generated in social networking sites and online newspapers. The new trend of data like images, audio, video, blogs, tweets, posts, shares \& likes, chats and many more falls under a new category of unstructured data. These varied type of data come under the 3rd 'V - Variety'. The last and 4th 'V - Veracity' is used to denote unstructured, unclean and uncertain data. The big data handles all the data which possess these Four 'V - Volume, Velocity, Variety and Veracity' as it's hard to handle dataset with these characteristics in the existing database systems.

To work with this volume of data requires distributing parts of the problem to multiple machines to handle in parallel. Whenever multiple machines are used in cooperation with one another, the probability of failures rises. In a single-machine environment, failure is not something that program designers explicitly worry about very often: if the machine has crashed, then there is no way for the program to recover anyway. This paper addresses the problem of data quality in electronic patient records using a computerized patient records report system with Apache HIVE and abstraction of Map reduce of big data technology.

\section{LITERATURE SURVEY}

The Existing Management System at Murab Hospital, Ilorin, Kwara, Nigeria. The information flow used is a one directional system where the receptionist refers patient to doctors, doctors referring patients to the pharmacist either in or out patients and the same way out. The system that is currently being used in the hospital is entirely manual. When a patient requests drugs from the staff, all the information is recorded manually from the drug dispenser (Pharmacist). 
Similarly when the supplier delivers drugs all the information from the dispenser to the account on drugs is recorded manually. The following are the weaknesses of the current system at the hospital:

1. The hospital staff finds it tiresome and time consuming when computing patient data, drug supplier and staff Payment receipts and voucher cards this leads to delay in medical reports.

2. The hospital Administration currently uses health record files for storing patients and drug supplier's

Information. This system of information storage is susceptible to security problems such as illegal modification and update of records.

3. The Staff usually waste a lot of time in retrieving data.

4. The paper work reduces the efficiency of the System.

In the existing system the data obtained from sensors can influence subsequent data analysis. The quality of the data plays an important role in outcome of the predictions made on the dataset. The data in an unstructured format may not pose an issue but inappropriate or erroneous data can lead to a faulty prediction or useless prediction. Let us analyze the critical data which will be a hindrance during analysis. Critical data may be missing values, data outliers, data drifting and communication errors. Generally, the failure or missing of sensor will create a missing value. There are many approaches for dealing with missing value. The most commonly used approach is replacing the missing value with ' 0 ' or ' 1 ' or skipping the missing value. Another approach is with replacing based on multivariate statistics of the data. Data outliers may be due to variability of data. For example, while calculating the average temperature of 50 sensors used in a room and 49 of them is between 20 and 25 degrees Celsius. But one sensor is at $175^{\circ} \mathrm{C}$. the median of data is will be between 20 and $25^{\circ} \mathrm{C}$. But the mean temperature will be between 35.5 and $40^{\circ} \mathrm{C}$. In this case, outlier detection algorithm is used. Data drift occurs during predictive analysis and machine learning. It means the drift of statistical properties of variable over time, which the model is trying to predict. There are two types - process drift and sensor drift. It is difficult to differentiate between these two drifts. Communication errors occur during transmission of data from sensor to base station and from base station to the server, error in storage, accessing error etc.

\section{III . EXSITING SYSTEM}

In existing system the footages of a vehicle at traffic signals are taken manually by a person or cops and then notices are send to person's residential address. There are so many cases where person claims that he/she has not crossed signal but the notice has come for example recently Traffic Police issuing ticket to traffic violator (pic: Supriya Khandekar) Sumit Chandra, an IT professional who travels from RT Nagar to Jayadeva Circle everyday says he received a notice for jumping a signal at MG Road on 7th March but he claims he never violated traffic rules, "I never jump signals and I remember not jumping a signal ever till date. I was shocked to receive this notice," he explains. These are just a few among many who claim to have received wrong notices for reasons such as driving over the speed limit, parking at no parking zones, jumping signals and so on. But the traffic police department denies any major flaws in issuing notices to the traffic violators. 


\section{PROPOSED SYSTEM}

Smart sensors are used for identifying drivers who ignore traffic laws. Smart sensors are activated when a driver passes through a certain point, usually located either in the middle of an intersection or at the end of the lane facing the red-light, when the traffic light is red. The Smart captures a snapshot of a vehicle. The sensor then sends the picture to a computer system to with the help of API's the plate number of a vehicle is extracted and then stored in a database for comparison if the a match is found, then automatically send a ticket and generated SMS bill to the vehicle owner's mobile and alert the authorities.

Red-light sensors can be placed in many different places and they can be so small that the driver does not even know that a sensor is in the area. Red-light sensors can be inserted into the traffic light itself, placed on top or below the traffic light, or placed on another object so that the driver does not know he is being watched. For example, some red-light sensors are placed in bushes or on nearby buildings. Others are placed where drivers can see them, which should prevent drivers from breaking the law by passing through the red light.

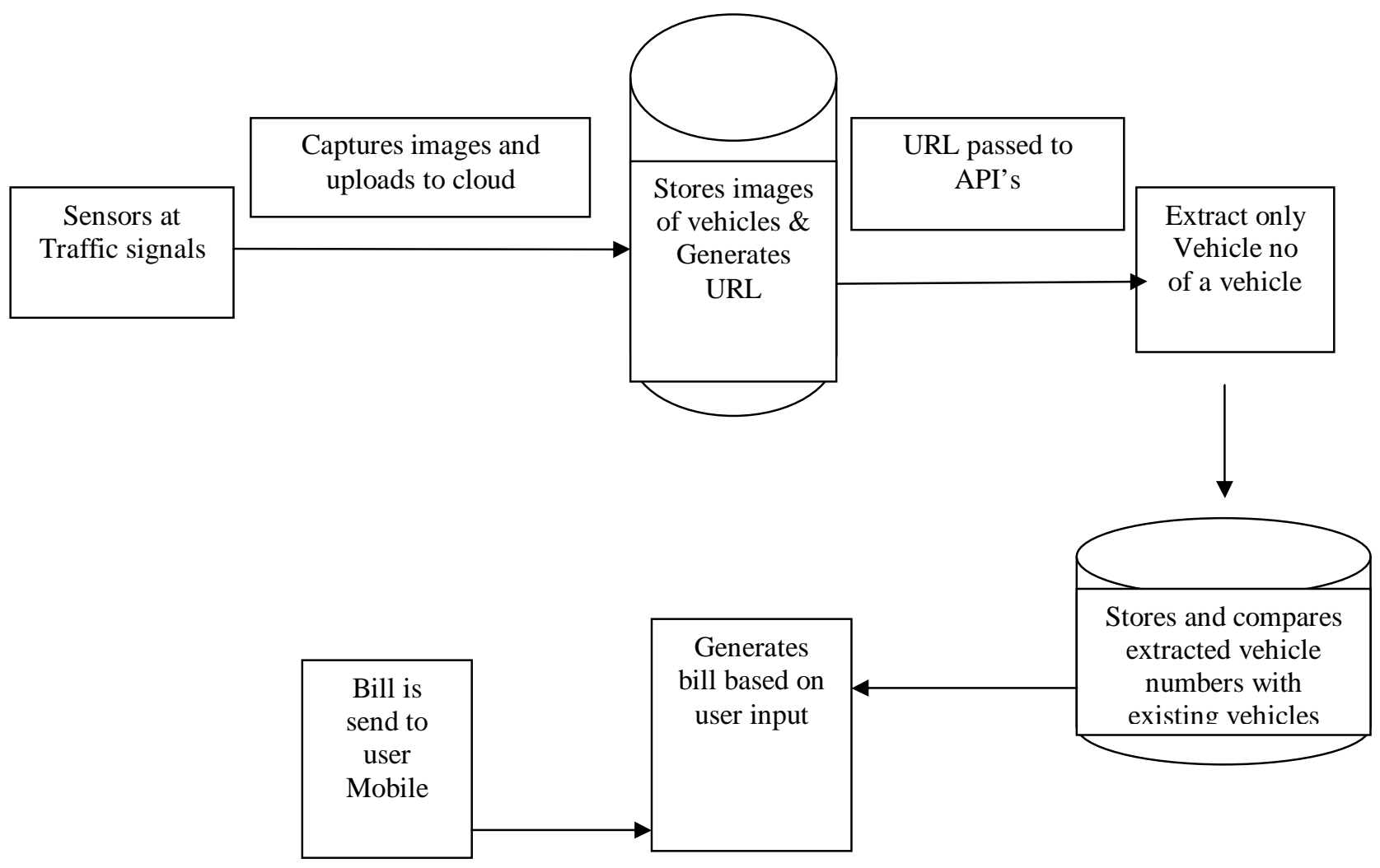

Fig:-1 System Architecture

MAP-REDUCE Algorithm: 
Map reduce works by breaking the processing into two phases. The map phase and the reduce phase. Each phase has key - value pairs as input and output, the types of which may be chosen by the programmer. The programmer also specifies two functions. The Map function and the reduce function.

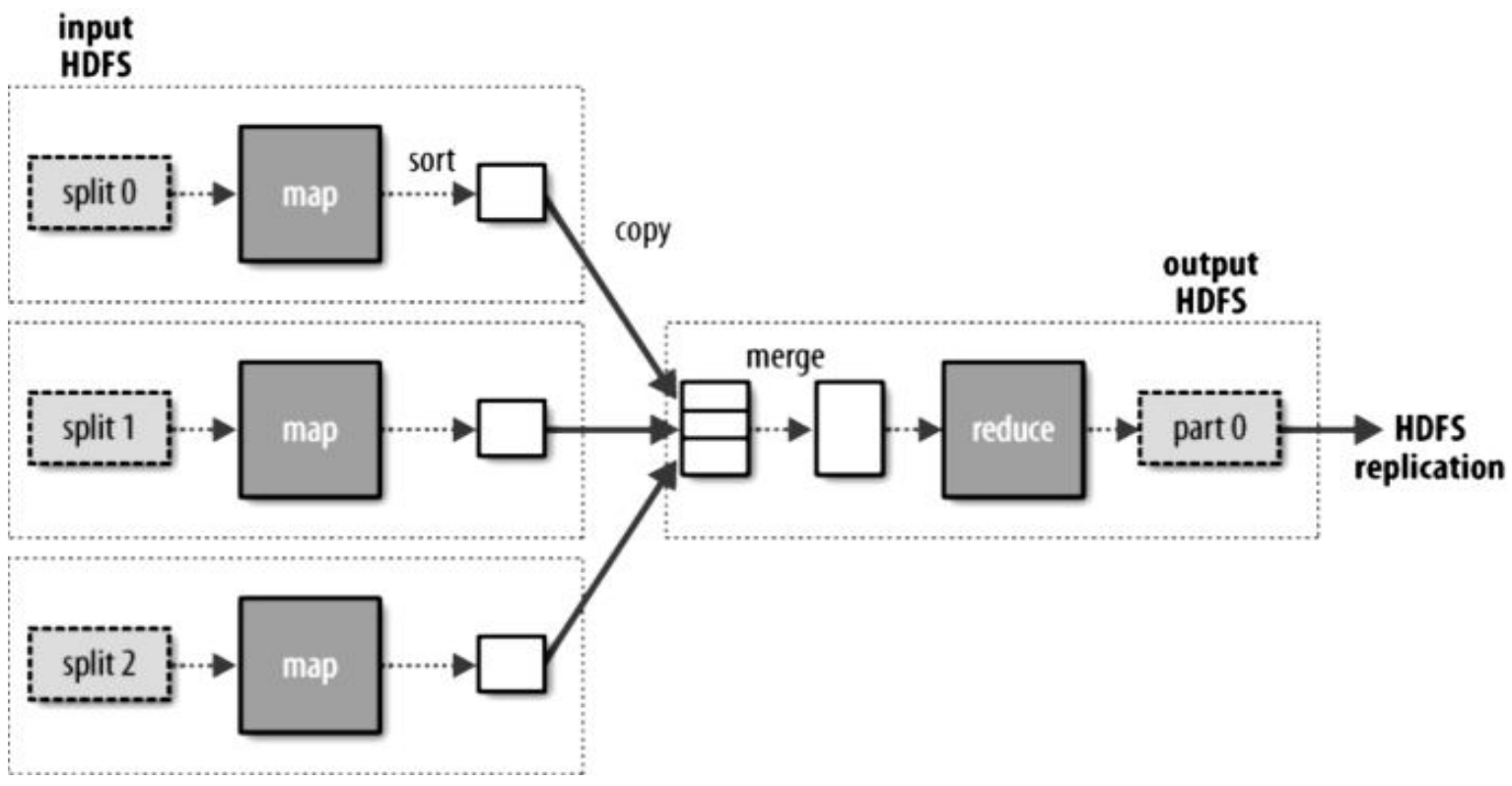

Fig - 2 MAP-REDUCE

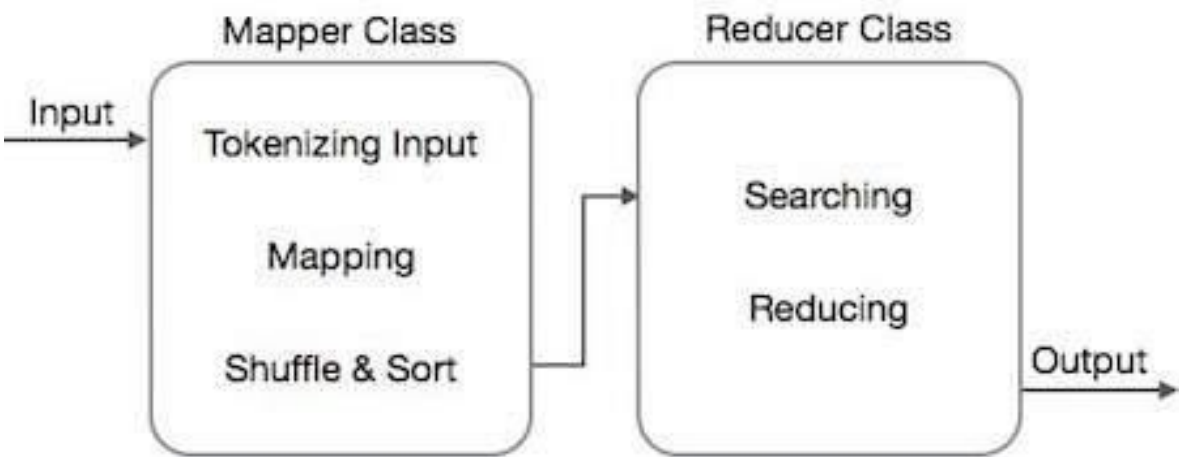

Map Reduce implements various mathematical algorithms to divide a task into small parts and assign them to multiple systems. In technical terms, MapReduce algorithm helps in sending the Map \& Reduce tasks to appropriate servers in a cluster.

These mathematical algorithms may include Sorting, Searching, Indexing and TF-IDF. Advantages:

1. An additional advantage of traffic cameras is that they can monitor intersections more efficiently and effectively than humans. 
2. If there is a traffic camera at an intersection, then there is no need for a police officer to be there, making it so that more officers are available for other duties

3. Traffic sensors provide $24 / 7$ traffic monitoring, so they can be present at intersections more than police

Officers and at a lower cost. And some believe traffic cameras to be safer since officers do not have to chase down offenders.

\section{V.CONCLUSION}

In this paper the main aim of our Advance Traffic Management System is to provide traffic management solutions. Our Advance Traffic Management System is a indigenously developed and designed specifically for Indian road conditions. The proposed plan can support the following applications like: Transportation monitoring: The system monitors safety driving rules like working or driving overtime, over speed or rash driving, braking, type slipping or skidding.

\section{REFERENCES}

[1] Vaclav' Jirkovsky a,b, Marek Obitko ${ }^{a}$, Petr Novak ${ }^{-b, c}$, and Petr Kadera ${ }^{b}$, "Big data analysis for sensor timeseries in automation", IEEE, 2014.

[2] TIRE PRESSURE MONITORING SYSTEM FMVSS No. 138, U.S. Department

[3] Xuejun Ding, Yong Tian, Member, IEEE, and Yan Yu, Member, IEEE, "A real-time big data gathering algorithm based on indoor wireless sensor networks for risk analysis of industrial operations", IEEE, 2015.

[4] Nicola Zingirian and Carlo Valenti, "Sensor clouds for intelligent truck monitoring", 2012 Intelligent Vehicles Symposium, Alcalá de Henares, Spain, June 3-7, 2012.

[5] Byoung Uk Kim, Chris Lynn, Neil Kunst a Tom Dudgeon, "Pattern analysis in real time with smart power sensors", IEEE, 2010.

[6] Jyoti R. Patole, "Clustering in wireless sensor network using K-means and Map reduce algorithm", Department of computer science engineering and information technology, college of engineering, Pune, 2012. 